\title{
Theoretical study on the mechanisms of ultrasonic modulation of multiply scattered light
}

Lihong V. Wang

Lihong V. Wang, "Theoretical study on the mechanisms of ultrasonic modulation of multiply scattered light," Proc. SPIE 4256, Biomedical Optoacoustics II, (15 June 2001); doi: 10.1117/12.429309

SPIE Event: BiOS 2001 The International Symposium on Biomedical Optics, 2001, San Jose, CA, United States 


\title{
Theoretical study on the mechanisms of ultrasonic modulation of multiply scattered light
}

\author{
Lihong V. Wang \\ Optical Imaging Laboratory, Biomedical Engineering Program \\ Texas A\&M University, College Station, Texas 77843-3120 \\ URL: http://oilab.tamu.edu; Email: LWang@ $\underline{\text { tamu.edu }}$
}

\begin{abstract}
An analytic model of the ultrasonic modulation of multiply scattered coherent light in scattering media is provided. The model is based on two mechanisms: the ultrasonic modulation of the index of refraction, which causes a modulation of the optical path lengths between consecutive scattering events, and the ultrasonic modulation of the displacements of Rayleigh scatterers, which causes a modulation of optical path lengths upon each scattering event. Multiply scattered light accumulates modulated optical path lengths along its path. Consequently, the intensity of the speckles that are formed by the multiply scattered light is modulated. In water solutions, for example, the contribution from the index of refraction is slightly less than the contribution from displacement when the scattering mean free path is less than a critical fraction of the acoustic wavelength, and it becomes increasingly greater than the contribution from displacement beyond this critical point.
\end{abstract}

Key words - ultrasonic modulation, optical speckles, multiple scattering, scattering media, random media, turbid media, wave propagation, coherent light, tissue optics, acousto-optics

\section{INTRODUCTION}

Ultrasound-modulated optical tomography is a new area of research for the imaging of scattering media, such as biological tissues. In this technology an ultrasonic wave is focused into a scattering medium to modulate light inside the medium. The advantage of this technology is its combination of optical contrast, which can reveal physiological information about biological tissues, and ultrasonic resolution, which is better than the optical resolution achievable by purely optical tomography in thick biological tissues. Marks et al. investigated the modulation of light in homogeneous scattering media with pulsed ultrasound. ${ }^{1}$ Wang et al. developed ultrasound-modulated optical tomography in scattering media with continuous-wave ultrasound. ${ }^{2}$ Kempe et al. showed experimentally the transition of ultrasonic modulation from the ballistic to the scattered regimes. ${ }^{3}$ Wang and Ku developed a frequency-swept technique to obtain scalable imaging resolution along the acoustic axis by frequency encoding of the light along the acoustic axis. ${ }^{4}$ Leveque et al. employed parallel detection of multiple speckles to improve signal-to-noise ratio, which was demonstrated with one-dimensional images of biological tissue. ${ }^{5}$ Yao and Wang further demonstrated this parallel-detection scheme with two-dimensional images of biological tissue. $^{6}$ In addition, by combining the parallel-detection and the frequency-swept techniques, Yao et al. obtained twodimensional images of biological tissues, in which one of the dimensions was along the acoustic axis. ${ }^{7}$ In place of transmission configurations, Lev et al. developed a reflection configuration for ultrasound-modulated optical tomography. ${ }^{8}$

Three possible mechanisms have been identified for the ultrasonic modulation of light in scattering media; which mechanism is dominant has, however, remained a mystery. The first mechanism is based on ultrasound-induced variations of the optical properties of the media. As an ultrasonic wave propagates in a scattering medium, the medium is compressed and rarified depending on location and time. Variations of density cause the optical properties of the medium-including the absorption coefficient, the scattering coefficient, and the index of refraction-to vary. Accordingly, the detected intensity of light varies with the ultrasonic wave. This mechanism, recently modeled by Mahan et al., ${ }^{9}$ does not require the use of a coherent light source. However, ultrasonic modulation of incoherent light has not been experimentally observed because it is too weak.

The second mechanism is based on variations of the optical phase in response to ultrasound-induced displacements of scatterers. The displacements of scatterers, assumed to follow ultrasonic amplitudes, modulate the physical path lengths of light traversing through the ultrasonic field. Multiply scattered light accumulates modulated physical path lengths along its path. Consequently, the intensity of the speckles formed by the multiply scattered light fluctuates with the ultrasonic wave. 
A theory given by Leutz and Mare ${ }^{10}$ modeled this mechanism but is valid only when the scattering mean free path is much greater than the acoustic wavelength.

The third mechanism is based on variations of the optical phase in response to ultrasonic modulation of the index of refraction. As a result of ultrasonic modulation of the index of refraction, the optical phase between two consecutive scattering events is modulated. Multiply scattered light accumulates modulated phases along its path. As in the second mechanism, the modulated phase causes the intensity of the speckles formed by the multiply scattered light to vary with the ultrasonic wave. This mechanism has never been modeled. Both the second and the third mechanisms require the use of coherent light.

In this paper, I present an analytic model based for the first time on both of the mechanisms for the ultrasonic modulation of coherent light. Further, I compare the relative contributions from the two mechanisms.

\section{MODEL}

A plane ultrasonic wave is assumed to insonify uniformly a homogenous scattering medium. The autocorrelation function of the electric field, $E(t)$, of the scattered light can be expressed as

$$
G_{1}(\tau)=C_{1} \int_{0}^{\infty} p(s)\left\langle E_{s}(t) E_{s}^{*}(t+\tau)\right\rangle d s
$$

where $C_{1}$ is a normalization factor such that $G_{1}(0)=1, E_{s}$ is the electric field of the scattered light of path length $s$, and $p(s)$ is the probability density function of $s$. The contributions to the autocorrelation function from Brownian motion and from the ultrasonic field are independent and can be treated separately. For conciseness, only the ultrasonic contribution is considered here.

The scattering medium, with a mean free path $l$, consists of noninteracting spherical Rayleigh scatterers. The ultrasonic contribution to the autocorrelation function by $s / l$ scattering events along path $s$ is

$$
\left\langle E_{s}(t) E_{s}^{*}(t+\tau)\right\rangle=\left\langle\exp \left\{-\mathrm{i}\left[\sum_{j=1}^{s / l+1} \Delta \phi_{n j}(t, \tau)+\sum_{j=1}^{s / l} \Delta \phi_{d j}(t, \tau)\right]\right\}\right\rangle,
$$

where $\Delta \phi_{n j}(t, \tau)=\phi_{n j}(t+\tau)-\phi_{n j}(t), \phi_{n j}$ is the phase variation induced by the modulated index of refraction along the $j$ th free path, $\Delta \phi_{d j}(t, \tau)=\phi_{d j}(t+\tau)-\phi_{d j}(t)$, and $\phi_{d j}$ is the phase variation induced by the modulated displacement of the $j$ th scatterer following the $j$ th free path. When the phase variations are much less than unity, the following approximation holds:

$$
\left\langle E_{s}(t) E_{s}^{*}(t+\tau)\right\rangle=\exp \left\{-\frac{1}{2}\left\langle\left[\sum_{j=1}^{s / l+1} \Delta \phi_{n j}(t, \tau)+\sum_{j=1}^{s / l} \Delta \phi_{d j}(t, \tau)\right]^{2}\right\rangle\right\} .
$$

On the one hand, the phase variation from the $j$ th free path is

$$
\phi_{n j}(t)=\int_{0}^{l_{j}} k_{0} \Delta n\left(\mathbf{r}_{j-1}, s_{j}, \theta_{j}, t\right) d s_{j},
$$

where $l_{j}$ is the length of the $j$ th free path, $k_{0}$ is the optical wave vector in vacuo, $\Delta n$ is the modulated index of refraction, $\mathbf{r}_{j}$ is the location of the $j$ th scatterer, $s_{j}$ is the distance along the $j$ th free path, and $\theta_{j}$ is the angle between the optical wave vector of the $j$ th free path and the acoustic wave vector $\mathbf{k}_{a}$.

The modulated index of refraction is

$$
\Delta n\left(\mathbf{r}_{j-1}, s_{j}, \theta, t\right)=n_{0} \eta k_{a} A \sin \left(\mathbf{k}_{a} \bullet \mathbf{r}_{j-1}+k_{a} s_{j} \cos \theta_{j}-\omega_{a} t\right),
$$

where $n_{0}$ is the background index of refraction; $k_{a}$ is the amplitude of $\mathbf{k}_{a} ; A$ is the acoustic amplitude; $\omega_{a}$ is the acoustic angular frequency; and $\eta$ is related to the adiabatic piezo-optical coefficient of the material $\partial n / \partial p$, the density $\rho$, the acoustic velocity $v_{a}: \eta=(\partial n / \partial p) \rho v_{a}^{2}$.

Completing the integration in Eq. (4), one obtains

$$
\phi_{n j}(t)=2 n_{0} k_{0} \eta A \sin \left(\mathbf{k}_{a} \bullet \mathbf{r}_{j-1}+k_{a} l_{j} \cos \theta_{j} / 2-\omega_{a} t\right) \sin \left(k_{a} l_{j} \cos \theta_{j} / 2\right) / \cos \theta_{j} .
$$

With some algebraic operations, one obtains 


$$
\left\langle\left[\sum_{j=1}^{s / l+1} \Delta \phi_{n j}(t, \tau)\right]^{2}\right\rangle=\left(\frac{s}{l}+1\right)\left(2 n_{0} k_{0} A\right)^{2} \delta_{n}\left[1-\cos \left(\omega_{a} \tau\right)\right]
$$

where

$$
\begin{gathered}
\delta_{n}=\left(\alpha_{n 1}+\alpha_{n 2}\right) \eta^{2}, \\
\alpha_{n 1}=k_{a} l \tan ^{-1}\left(k_{a} l\right) / 2, \\
\alpha_{n 2}=2 \beta^{2} /(1-\chi), \\
\beta=\pi / 4-1 /\left(2 k_{a} l\right)\left[{ }_{2} F_{1}\left(1,1 / 2,3 / 2,-1 /\left(k_{a} l\right)^{2}\right)\right], \\
\chi=\tan ^{-1}\left(k_{a} l\right) /\left(k_{a} l\right),
\end{gathered}
$$

where ${ }_{2} F_{1}()$ is the generalized hypergeometric function. ${ }^{11}$

On the other hand, the phase variation from the $j$ th scattering event is

$$
\phi_{d j}(t)=-n_{0} k_{0}\left(\hat{\mathbf{k}}_{j+1}-\hat{\mathbf{k}}_{j}\right) \bullet \mathbf{A} \sin \left(\mathbf{k}_{a} \bullet \mathbf{r}_{j}-\omega_{a} t\right),
$$

where $\hat{\mathbf{k}}_{j}$ is the unit optical wave vector for the $j$ th free path, and $\mathbf{A}$ is the acoustic-amplitude vector. The following variance of phase variation can be derived similarly as for Eq. (7):

$$
\left\langle\left[\sum_{j=1}^{s / l} \Delta \phi_{d j}(t, \tau)\right]^{2}\right\rangle=\frac{s}{l}\left(2 n_{0} k_{0} A\right)^{2} \delta_{d}\left[1-\cos \left(\omega_{a} \tau\right)\right]
$$

where $\delta_{d}=1 / 6$; and the following relation for Rayleigh scattering is used in the derivation: $\left\langle\left[\left(\hat{\mathbf{k}}_{j+1}-\hat{\mathbf{k}}_{j}\right) \bullet \mathbf{A}\right]^{2}\right\rangle=(1 / 3)\left\langle\left(\hat{\mathbf{k}}_{j+1}-\hat{\mathbf{k}}_{j}\right)^{2}\right\rangle A^{2}=2 A^{2} / 3$.

The autocorrelation function in Eq. (1) becomes

$$
G_{1}(\tau)=C_{1} \int_{0}^{\infty} p(s) \exp \left(-(2 s / l)\left(\delta_{n}+\delta_{d}\right)\left(n_{0} k_{0} A\right)^{2}\left[1-\cos \left(\omega_{a} \tau\right)\right]\right) d s
$$

in the diffusion limit, where the independence between $\Delta \phi_{n j}$ and $\Delta \phi_{d k}$ is used. A coherent optical beam from an extended plane source is assumed to be incident upon a slab of thickness $L$, and the transmitted light is detected from a point. The function $p(s)$ is given by diffusion theory with a zero-boundary condition. ${ }^{12}$ Carrying out the integration in Eq. (15), ${ }^{13}$ one obtains

$$
G_{1}(\tau)=\frac{(L / l) \sinh \left[\left\{\varepsilon\left[1-\cos \left(\omega_{a} \tau\right)\right]\right\}^{1 / 2}\right]}{\sinh \left[(L / l)\left\{\varepsilon\left[1-\cos \left(\omega_{a} \tau\right)\right]\right\}^{1 / 2}\right]}
$$

where $\varepsilon=6\left(\delta_{n}+\delta_{d}\right)\left(n_{0} k_{0} A\right)^{2}$.

The intensity of the modulated speckles at frequency $n \omega_{a}$ can be calculated based on the Wiener-Khinchin theorem: $I_{n}=T_{a} \int_{0}^{T_{a}} \cos \left(n \omega_{a} \tau\right) G_{1}(\tau) d \tau$, where $T_{a}$ is the acoustic period. The modulation depth, $M$, is defined as the ratio between the intensity at the fundamental frequency $I_{1}$ and the unmodulated intensity $I_{0}$.

\section{NUMERICAL RESULTS}

Figure 1(a) shows the modulation depth as a function $k_{a} l$ with a constant $l$ contributed from the index of refraction alone, $M_{n}$; the modulation depth contributed from displacement alone, $M_{d}$; and the modulation depth contributed from both, $M_{\text {sum }}$. The symbols are from an independent Monte Carlo simulation accomplished by programming Eqs. (1) and (2) into a public-domain software package for light transport in scattering media. ${ }^{14}$ The analytic model presented here agrees with the Monte Carlo model very well. A plot of the ratio between $M_{n}$ and $M_{d}$ is shown in Fig. 1(b). The contribution from the index 
of refraction increases with $k_{a} l$ because a shorter acoustic wavelength increases the accumulation of phase modulation in each free path. By contrast, the contribution from displacement stays constant, independent of $k_{a}$ and $l$. As a result, the ratio between $M_{n}$ and $M_{d}$ increases with $k_{a} l$ and crosses a critical point at $k_{a} l=0.559$ or $l=0.0890 \lambda_{a}$, where the ratio is unity. When $k_{a} l$ is less than this critical point, the contribution from the index of refraction is slightly less than the contribution from displacement; beyond this critical point, the contribution from the index of refraction increases and can significantly outmatch the contribution from displacement.
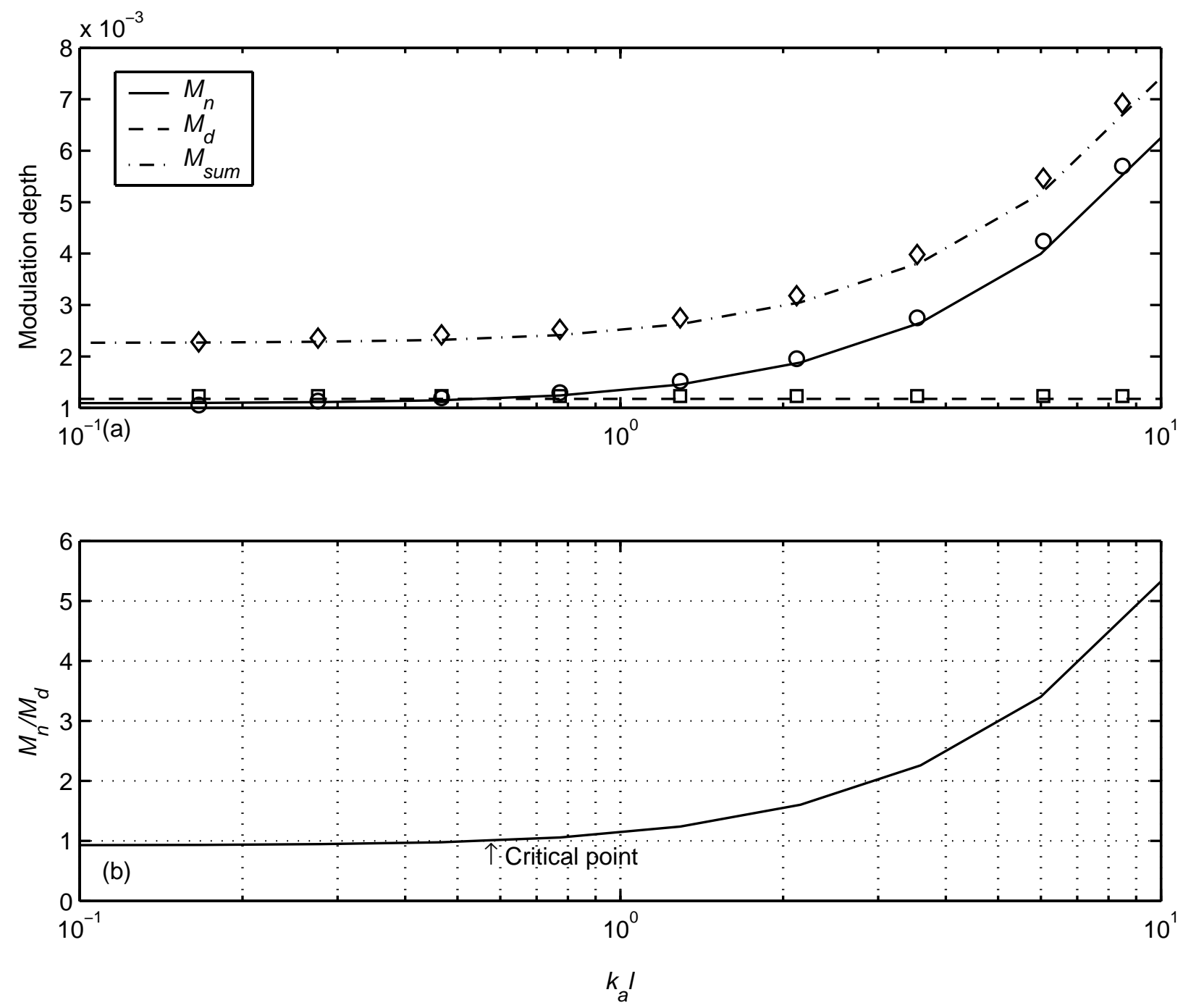

Fig. 1. (a) The modulation depths: $M_{n}, M_{d}$, and $M_{\text {sum }}$ and (b) the ratio between $M_{n}$ and $M_{d}$ as a function $k_{a} l$ with a constant $l=$ $1 \mathrm{~mm}$. The following parameters are used: $\partial n / \partial p=1.466 \times 10^{-10} \mathrm{~m}^{2} / \mathrm{N}, \rho=1000 \mathrm{~kg} / \mathrm{m}^{3}, v_{a}=1480 \mathrm{~m} / \mathrm{s}, \omega_{a}=2 \pi \mathrm{MHz}$, $n_{0}=1.33, \lambda_{0}=500 \mathrm{~nm}, L=5 \mathrm{~cm}, A=0.1 \mathrm{~nm}$.

\section{SUMMARY}

In summary, an analytic model of the ultrasonic modulation of multiply scattered coherent light in scattering media is provided. The model is based on two mechanisms: the ultrasonic modulation of the index of refraction, which causes a modulation of the optical path lengths between consecutive scattering events, and the ultrasonic modulation of the displacements of Rayleigh scatterers, which causes a modulation of optical path lengths upon each scattering event. Multiply scattered light accumulates modulated optical path lengths along its path. Consequently, the intensity of the speckles that are formed by the multiply scattered light is modulated. In water solutions, for example, the contribution from the index of 
refraction is slightly less than the contribution from displacement when the scattering mean free path is less than a critical fraction of the acoustic wavelength, and it becomes increasingly greater than the contribution from displacement beyond this critical point. This analytic model agrees well with an independent Monte Carlo model. Both of the mechanisms are coherent phenomena, requiring the use of a coherent light source. This work clarifies the mystery about the mechanisms of ultrasonic modulation of light in scattering media.

\section{ACKNOWLEDGMENTS}

This project was sponsored in part by National Science Foundation grant BES-9734491, Texas Higher Education Coordinating Board grant 000512-0123-1999, and National Institutes of Health grants R29 CA68562 and R01 CA71980.

\section{REFERENCES}

1. F. A. Marks, H. W. Tomlinson, and G. W. Brooksby, Proc. Soc. Photo-Opt. Instrum. Eng. 1888, 500 (1993).

2. L.-H. Wang, S. L. Jacques, and X. Zhao, Opt. Lett. 20, 629 (1995).

3. M. Kempe, M. Larionov, D. Zaslavsky, and A. Z. Genack, J. Opt. Soc. Am. 14, 1151 (1997).

4. L.-H. V. Wang and G. Ku, Opt. Lett. 23, 975 (1998).

5. S. Leveque, A. C. Boccara, M. Lebec, and H. Saint-Jalmes, Opt. Lett. 24, 181 (1999).

6. G. Yao and L.-H. V. Wang, Appl. Opt. 39, 659 (2000).

7. G. Yao, S.-L. Jiao, and L.-H. V. Wang, Opt. Lett. 25, 734 (2000).

8. A. Lev, Z. Kotler, and B. G. Sfez, Opt. Lett. 25, 378 (2000).

9. G. D. Mahan, W. E. Engler, J. J. Tiemann, and E. Uzgiris, Proc. Natl. Acad. Sci. USA 95, 14015 (1998).

10. W. Leutz and G. Maret, Physica B 204, 14 (1995).

11. G. B. Arfken and H. J. Weber, Mathematical Methods for Physicists, 4th ed. (Academic Press, San Diego, CA, 1995).

12. M. S. Patterson, B. Chance, and B. C. Wilson, Appl. Opt. 28, 2331 (1989).

13. D. J. Pine, D. A. Weitz, P. M. Chaikin, and E. Herbolzheimer, Phys. Rev. Lett. 60, 1134 (1988).

14. L.-H. Wang, S. L. Jacques, and L.-Q. Zheng, Comp. Meth. Prog. in Biomed. 47, 131 (1995). Software available at URL: http://oilab.tamu.edu. 